\title{
Brain Tumor Detection Based on Multilevel 2D Histogram Image Segmentation Using DEWO Optimization Algorithm
}

\author{
Sumit Kumar, Amity University, India \\ Garima Vig, Amity University, India \\ (iD) https://orcid.org/0000-0002-8584-8953 \\ Sapna Varshney, University of Delhi, India \\ Priti Bansal, Netaji Subhas University of Technology, India
}

\begin{abstract}
Brain tumor detection from magnetic resonance (MR)images is a tedious task but vital for early prediction of the disease which until now is solely based on the experience of medical practitioners. Multilevel image segmentation is a computationally simple and efficient approach for segmenting brain MR images. Conventional image segmentation does not consider the spatial correlation of image pixels and lacks better post-filtering efficiency. This study presents a Renyi entropy-based multilevel image segmentation approach using a combination of differential evolution and whale optimization algorithms (DEWO) to detect brain tumors. Further, to validate the efficiency of the proposed hybrid algorithm, it is compared with some prominent metaheuristic algorithms in recent past using between-class variance and the Tsallis entropy functions. The proposed hybrid algorithm for image segmentation is able to achieve better results than all the other metaheuristic algorithms in every entropy-based segmentation performed on brain MR images.
\end{abstract}

\section{KEYWORDS}

2D Histogram, Between-Class Variance, Brain MR Image Segmentation, Multilevel Thresholding, Whale Optimization

\section{INTRODUCTION}

In modern era, clinical experts take help of e-healthcare and automated systems to provide better diagnosis to the patients. Inspection of abnormalities in internal organs is a tedious job that require invasive/non-invasive imaging approach. These abnormalities are extensively recorded using magnetic resonance (MR) imaging, after which the images are processed to infer the location and severity of disease in the internal organs. In healthcare domain, image examination is an integral procedure where pre-processing, investigation, categorization and post-processing of medical images is performed. Medical image segmentation is the widely adopted technique in many real time applications as the pixel gray level value for objects in an image and the pixel gray level value for the image background are substantially different. This can be exploited to get various homogeneous regions in a medical image for synthesis and analysis (Heimann et al., 2009). Image segmentation can be performed by various methods such as threshold, edge detection, region growing, split and merge, neural networks,

This article, originally published under IGI Global's copyright on July 1, 2020 will proceed with publication as an Open Access article starting on January 25, 2021 in the gold Open Access journal, International Journal of E-Health and Medical Communications (converted to gold Open Access January 1, 2021), and will be distributed under the terms of the Creative Commons Attribution License (http://creativecommons.org/licenses/by/4.0/) which permits unrestricted use, distribution, and production in any medium, provided the author of the original work and original publication source are properly credited. 
clustering, compression and histogram techniques. Thresholding techniques extract objects based on the gray level of pixels by differentiating objects from other objects and background in an image (Sezgin \& Sankur, 2004). Some of the prominent domains where thresholding can be applied are: medical imaging applications like cell images (Sieracki et al., 1989), x-ray computed tomography (Oh \& Lindquist, 1999), con-focal microscopy using laser scanners (Jubany et al., 2009), Cancer and tumor prediction (Al-Tarawneh, 2012), etc. Multilevel thresholding technique is one of the prominent approaches of image segmentation as it extracts more than one region of interest in a simple and efficient manner. Some of the criterion functions that are optimized in thresholding are class variance (Otsu, 1979), classification error (Kittler \& Illingworth, 1986), or entropy (Pun, 1980). Some of the prominent entropy thresholding functions include Kapur's entropy (Wong \& Sahoo, 1989), Renyi's entropy (Sahoo \& Arora, 2004), Tsallis entropy (Tang et al., 2009), and Otsu's thresholding method (Otsu, 1979). The major drawback of entropy-based thresholding functions is that they only use the gray level distribution of an image resulting in same histogram and thresholds for different images. This drawback was addressed by Abutaleb (1989), by developing the concept of a 2D histogram that contains spatial information in addition to gray level distribution information. 2D histogram-based techniques ignore information related to edges and only consider objects and background information. Non-local means based 2D histogram techniques are provided with better post filter clarity of images with better results (Mittal \& Saraswat, 2018). Conventional multilevel thresholding techniques require exhaustive computation and hence are time-consuming (Kapur et al., 1985). This drawback is addressed by using threshold level as a spatial dimension for metaheuristic algorithms. Over the last few years, many researchers have shown interest in solving multilevel thresholding segmentation problem using different metaheuristic algorithms like Simulated Annealing (SA) (Fengjie et al., 2009), Ant Colony Optimization(ACO) (Wang et al., 2005), Genetic Algorithm(GA) (Maulik, 2009), Firefly Algorithm (FA) (Xiao-Feng et al., 2016), Artificial Fish-Swarm (AFS) (Xiao-Feng et al., 2016), Swallow Swarm Optimization (SSO) (Panda et al., 2017), Bacterial Foraging Algorithm (BFA) (Sathya \& Kavalvizhi, 2011), Differential Evolution (DE) (Sarkar \& Das, 2013) etc. In this study, a new hybrid algorithm DEWO, which is a combination of Differential Evolution and Whale Optimization (WO) algorithms is proposed for brain tumor detection using an efficient 2D histogram multilevel thresholding technique based on non-local means filter and Renyi entropy. Results obtained from the proposed hybrid algorithm has been compared with some prominent metaheuristic algorithms like Cuckoo Search (CS), DE, Artificial Bee Colony (ABC), and WO. Further, the proposed hybrid algorithm is analyzed for different entropy functions like Tsallis entropy and between-class variance. The brain MR images used in this study for segmentation are taken from the Reference Image Database to Evaluate Therapy Response (RIDER) Neuro MRI project data, accessed from The Cancer Imaging Archive (TCIA).

The remainder of the paper is organized in the following sections: section 2 elaborates related work, section 3 gives a brief description of the methodology, section 4 elaborates experimental result of the proposed hybrid algorithm and comparison with other metaheuristic algorithms and entropy functions and section 5 concludes the study.

\section{LITERATURE REVIEW}

Medical image segmentation for detection of abnormalities from medical imaging modalities is a very important process for deciding right therapy at the right time. Many techniques have been adopted by researches for medical image analysis. Some of the recent researches are discussed below.

Abdeldaim et al. (2018) developed a computer aided system for the diagnosis of acute lymphoblastic leukemia. White blood cell segmentation act as a crucial step of diagnosis classification, where segmentation is performed by calculating roundness and solidity of white blood cells using convex hull area of the white blood cells. Yeo et al. (2018) developed a statistical model for the segmentation of biomedical images like MRI images. Segmentation factorizes the statistical model into different shapes and poses for estimating the shape of subcutaneous adipose tissue. Mohammed 
et al. (2017) detected Nasopharyngeal Carcinoma in medical images using region growing based thresholding technique that uses iterative threshold selection by calculating every neighborhood less values from image histogram within the surroundings of frontage. Kumar et al. (2012) developed a hybrid technique based on modified ACO and 2D Otsu's method of image thresholding to get efficient results for the segmentation of CT scan images of lungs. Saleh and Eswaran (2012) developed an approach for the extraction of retinal images where contrast enhancement, background removal and H-maxima transformations are used for preprocessing of images and multilevel thresholding and binarization are performed for retinal image extraction. Lee et al. (2015) analyzed thresholding, region growing, clustering, ANN and level set methods of image segmentation for different types of medical images like CT scan images, 3D MRI, MRI, Ultrasound and X-ray images to characterize and summarize best methods for every type of medical images. Li et al. (2015) performed stomach segmentation using dynamic-context cooperative quantum PSO and Otsu segmentation with enhanced exploration and exploitation of the search space. Namburua et al. (2017) performed an efficient brain image segmentation based on soft fuzzy rough c-means set that helped in effective utilization of histograms by converging fast and narrowing probability of occurrence of mistakes. Bahadure et al. (2017) performed brain image segmentation using Berkeley Wavelet Transformation (BWT) that helped to reduce the complexity of the segmentation process and Support Vector Machine (SVM) to classify brain tumor images. SVM increases the accuracy of classification result when used with BWT and feature extraction mechanism. Rajinikanth et al. (2017) performed a comparative analysis of three entropy functions namely Kapur's entropy, Shannon entropy, and Tsallis entropy-based image segmentation of brain MR images using Teaching Learning based optimization algorithm. They found that Shannon entropy performed better than other entropic functions; then they applied level set method for extraction of glioma section of brain MR images. Panda et al. (2017) developed an adaptive Swallow Swarm Optimization (SSO) technique along with Evolutionary Gray Gradient Algorithm (EGGA) for brain MR images containing many regions of interest. Nakib et al. (2007) developed a Particle Swam Optimization (PSO)-based thresholding technique for segmentation of brain MR images using 2D survival exponential entropy to get better results from conventional 2D survival exponential entropy-based segmentation. Shahdoosti and Tabatabaei (2019) developed a region-based segmentation technique for the fusion of medical images to reduce the sensitivity of MR images towards noise and further applied ACO-based clustering algorithm to get efficient results for segmentation. Sumathi et al. (2018) presented Cuckoo Search (CS-based image segmentation technique using Kapur's entropy for tumor extraction from brain and breast MR Images to provide a good resistance towards noise alteration in images. Sarkar et al. (2017) developed a segmentation approach combining Renyi and cross-entropy for addressing pareto optimal multi-objective nature of natural and medical images using decomposition-based Multi-Objective Evolutional Algorithm variant of Differential Evolution (MOEA/D-DE) to efficiently segment lesion regions of brain tumor. Zhu et al. (2017) developed a method of liver cyst extraction using Wellner's thresholding algorithm and PSO to get better results as liver ultrasound images have low signal-to-noise-ratio and low contrast. Raja et al. (2018) presented a region growing approach of image segmentation using a combination of Bat Algorithm (BA) and Tsallis entropy for evaluating contrast improved T1 modality-based brain and breast MR images in an efficient manner. Roopini et al. (2018) developed an approach for multilevel thresholding image segmentation based on Firefly Algorithm and fuzzy entropy in combination with distance regularized level set to extract tumor from the brain MR images. Zhang et al. (2017) developed a multi-scale 3D Otsu thresholding method for precise brain MR image segmentation. Dawngliana et al. (2015) presented a hybrid method of multilevel thresholding and level set techniques to get refined segmented images for boundary variation. Table 1 summarizes the recent related work done by researchers. 


\section{PRELIMINARIES}

This section presents the overview of techniques and concepts employed in this study.

\section{Table 1. Literature review summarization}

\begin{tabular}{|c|c|c|}
\hline Author(s) & Application area & Methodology \\
\hline Abdeldaim et al. (2018) & $\begin{array}{l}\text { Acute Lymphoblastic Leukemia } \\
\text { diagnosis }\end{array}$ & $\begin{array}{l}\text { Computer aided system for } \\
\text { segmentation using convex hull }\end{array}$ \\
\hline Yeo et al. (2018) & Biomedical Imaging & Statistical model for segmentation \\
\hline Mohammed et al. (2017) & Nasopharyngeal Carcinoma Imaging & Region growing based thresholding \\
\hline Kumar et al. (2012) & Lung CT Scan Imaging & Modified ACO with 2D Otsu method \\
\hline Saleh and Eswaran (2012) & Retinal Imaging & $\begin{array}{l}\text { H-maxima transformation, multilevel } \\
\text { thresholding and binarization }\end{array}$ \\
\hline Lee et al. (2015) & Medical Imaging & Survey Paper \\
\hline Li et al. (2015) & Stomach CT Scan Imaging & $\begin{array}{l}\text { Dynamic-Context Cooperative } \\
\text { Quantum PSO and Otsu segmentation }\end{array}$ \\
\hline Namburua et al. (2017) & \multirow{5}{*}{ Brain MR Imaging } & Soft fuzzy rough c-means \\
\hline Bahadure et al. (2017) & & $\begin{array}{l}\text { Berkeley wavelet Transformation } \\
\text { (BWT) and Support Vector Machine } \\
\text { (SVM) }\end{array}$ \\
\hline Rajinikanth et al. (2017) & & $\begin{array}{l}\text { Kapur's entropy, Shannon entropy, } \\
\text { and Tsallis entropy and using } \\
\text { Teaching Learning based optimization } \\
\text { algorithm }\end{array}$ \\
\hline Panda et al. (2017) & & $\begin{array}{l}\text { Adaptive Swallow Swarm } \\
\text { Optimization (SSO) and Evolutionary } \\
\text { Gray Gradient Algorithm (EGGA) }\end{array}$ \\
\hline Nakibet al. (2007) & & $\begin{array}{l}\text { PSO and 2D survival exponential } \\
\text { entropy }\end{array}$ \\
\hline Shahdoosti and Tabatabaei (2019) & MR Imaging & ACO based Clustering \\
\hline Sumathi et al. (2018) & Brain and Breast MR Imaging & CS algorithm using Kapur's entropy \\
\hline Sarkar et al. (2017) & Medical Imaging & $\begin{array}{l}\text { Decomposition based Multi-Objective } \\
\text { Evolutional Algorithm variant of } \\
\text { Differential Evolution (MOEA/D- } \\
\text { DE) and Hybrid of Renyi entropy and } \\
\text { cross-entropy. }\end{array}$ \\
\hline Zhu et al. (2017) & Ultrasound Imaging & $\begin{array}{l}\text { Wellner's thresholding algorithm and } \\
\text { PSO }\end{array}$ \\
\hline Raja et al. (2018) & Brain and Breast MR Imaging & $\begin{array}{l}\text { Region growing segmentation using } \\
\text { Bat Algorithm and Tsallis entropy }\end{array}$ \\
\hline Roopini et al. (2018) & \multirow{3}{*}{ Brain MR Imaging } & $\begin{array}{l}\text { Multilevel thresholding based on } \\
\text { Firefly Algorithm using fuzzy entropy } \\
\text { and distance regularized level set }\end{array}$ \\
\hline Zhang et al. (2017) & & Multi-scale 3D Otsu thresholding \\
\hline Dawngliana et al. (2015) & & $\begin{array}{l}\text { Hybrid technique of multilevel } \\
\text { thresholding and level set method. }\end{array}$ \\
\hline
\end{tabular}




\section{A. Renyi Entropy:}

Entropy is the measure of global amount of information contained in an image histogram, as stated by Shannon in his initiated research work known as information theory. Renyi entropy is a generalization of Shannon entropy proposed by Renyi. Renyi entropy is a more adaptable and flexible function due to its dependence on parameters. It is an extended version of Shannon entropy which applied to continuous family of entropy measures. Renyi entropy can be stated as:

$\mathrm{R}_{\mathrm{q}}(\mathrm{I})=\frac{1}{1-\mathrm{q}} \ln \sum_{\mathrm{i}=1}^{\mathrm{E}} \mathrm{p}_{\mathrm{i}}^{\mathrm{q}}$

where:

$\mathrm{R}_{\mathrm{q}}$ : Renyi entropy for entropy index $\mathrm{q}$

$\mathrm{I}$ : Discrete random variable with possible outcomes 1 to $\mathrm{E}$

q: Entropy index

$\mathrm{p}_{\mathrm{i}}$ : Probability of discrete variable for $\mathrm{i}=1$ to $\mathrm{E}$

Shannon entropy is a special case of Renyi entropy where $q \rightarrow 1$. Here $q$ is known as order of entropy.

B. Non- local means filter:

Non local helps get better post filter clarity by including edge information in the right amount. This method calculates means of all pixels in an image by performing weighted comparison of each centre pixel of filter with the target pixel. Consider $\mathrm{X}(\mathrm{u})$ and $\mathrm{X}(\mathrm{v})$ as two pixels of input image then non local means output image pixels can be computed as:

$$
\mathrm{Y}(\mathrm{u})=\frac{\sum_{\mathrm{v} \in \mathrm{X}} \mathrm{X}(\mathrm{v}) \cdot \mathrm{w}(\mathrm{u}, \mathrm{v})}{\sum_{\mathrm{v} \in \mathrm{X}} \mathrm{w}(\mathrm{u}, \mathrm{v})}
$$

where $\mathrm{w}(\mathrm{u}, \mathrm{v})=\mathrm{e}^{-\frac{|\mathrm{H} / \mathrm{u}(\mathrm{u})-\mathrm{s} / \mathrm{v} v|^{2}}{\tilde{\mathrm{A}}(\mathrm{u}) \cdot \tilde{\mathrm{A}}(\mathrm{v})}}$ here $\mu$ is mean and $\sigma$ is the standard deviation for the pixels corresponding to the filter mask of size $m \times m$.

\section{2-D Histogram:}

$$
g(x, y)(x, y) \mathrm{H}(\mathrm{i}, \mathrm{j})=\mathrm{O}_{\mathrm{ij}} f(x, y) f(x, y) g(x, y) O_{i j} \mathrm{i}, \mathrm{ji}=\mathrm{f}(\mathrm{x}, \mathrm{y}) \mathrm{j}=\mathrm{g}(\mathrm{x}, \mathrm{y}) M \times N 2 \mathrm{D}
$$

Histogram for an image is obtained by mapping non local means image pixel with gray scale image pixels to provide better exploitation of spatial information of image. Consider for spatial coordinate $(x, y)$.of pixel of image $f(x, y)$.represents pixel value of gray scale image and $g(x, y)$.represent pixel of non local mean filtered image. 2D histogram matrix value can be calculated by mapping the occurrence of $f(x, y)$.with $g(x, y)$. Mathematically,

$\mathrm{H}(\mathrm{i}, \mathrm{j})=\mathrm{O}_{\mathrm{ij}}$. where $O_{i j}$.is the total number of occurrence of pair $\mathrm{i}, \mathrm{j} \cdot ; \mathrm{i}=\mathrm{f}(\mathrm{x}, \mathrm{y})$. and $\mathrm{j}=\mathrm{g}(\mathrm{x}, \mathrm{y})$. Normalized histogram is calculated by dividing occurrence value with size of the image $M \times N$. Mathematically, 
$\mathrm{N}(\mathrm{i}, \mathrm{j})=\mathrm{O}_{\mathrm{ij}} / \mathrm{M} \times \mathrm{N}$. Histogram depicted in the table show that object and background information can be obtained through diagonal elements, hence only diagonal elements are considered for further obtaining optimal threshold values.

D. Differential Evolution:

DE is an evolutionary optimisation technique developed by Storn and Price in 1997. DE is a population-based search technique that help to achieve robust optimum solution for a given problem faster than other evolutionary problems. DE performs mutation and crossover by mixing mutated vector generated with some predefined vector, called target vector. The vector generated after crossover is the trial vector. In the selection process, decision about inclusion of trial vector in the next generation is taken using greedy criteria; then the target vector is replaced with the trial vector if latter is strictly superior.

Mutation: $\mathrm{V}=\mathrm{X}_{\mathrm{r} 1}+\mathrm{F}^{*}\left(\mathrm{X}_{\mathrm{r} 2}-\mathrm{X}_{\mathrm{r} 3}\right)$

Crossover: $\mathrm{U}= \begin{cases}V & \text { if } \text { rand }_{\mathrm{j}} \leq C r \| \mathrm{j}_{\mathrm{rand}}=j \\ X & \text { otherwise }\end{cases}$

Selection: $\mathrm{X}(\mathrm{t}+1)= \begin{cases}U & \text { iff }(\mathrm{U}) \leq f(\mathrm{X}) \\ X & \text { otherwise }\end{cases}$

where:

- $\mathrm{X}_{\mathrm{r} 1}, \mathrm{X}_{\mathrm{r} 2}, \mathrm{X}_{\mathrm{r} 3}$ : Three different random individuals

- V: Mutated vector

- F: Scaling factor

- Cr: Crossover rate

- $\mathrm{U}$ : Trial vector

- $\mathrm{X}(\mathrm{t}+1)$ : Individual for generation $\mathrm{t}+1$

E Whale Optimization (WO):

WO was proposed by Mirjalili and Lewis in 2016. WO is a population-based algorithm that exploit hunting method of humpback whales known as bubble net feeding technique. Whales create a spiral shaped bubble path. Exploitation of the search space is performed using bubble net method which constitute of two mechanism: shrinking encircling mechanism and spiral updating position. Whales move around prey in a spiral shaped thereby shrinking circular path simultaneously for perfect attack. In WO, a probability of 50\% is assigned to both paths for every position update. WO consists of minimum number of heuristics and internal parameter and is computationally a simple algorithm.

Position Update: $X(t+1)= \begin{cases}X^{*}(t)-A . D & \text { if } p<0.5 \\ D^{\prime} \cdot e^{b l} \cdot \cos (2 \pi l)+X^{*}(t) & \text { if } p \geq 0.5\end{cases}$

where:

- $\quad \mathrm{D}=\left|\mathrm{C} . \mathrm{X}^{*}(\mathrm{t})-\mathrm{X}(\mathrm{t})\right|$

- $\mathrm{X}^{*}(\mathrm{t})$ : position of best solution

- $\mathrm{A}=2 \mathrm{a} \cdot \mathrm{r}-\mathrm{a} \cdot \mathrm{C}=2 \mathrm{r}$.

- A and C: coefficient vectors; a: linearly decreasing vector from 2 to 0 ; r: random vector

- $\quad D^{\prime}=\left|X^{*}(t)-X(t)\right|$ i.e. distance of current solution from best solution obtained so far

Metaheuristic hybridization is the recent trend in the field of optimization; achieved by combining one metaheuristic algorithm with complementing metaheuristic algorithm. Differential Evolution is an efficient algorithm used for solving many classical optimization problems. DE covers the 
entire search space of the algorithm in an effective manner. Best/DE/1 variant of DE can achieve good exploitation of the input search space by using the best individual obtained do far. However, optimum result of DE is sensitive to the choice of scaling factor $(\mathrm{F})$ and crossover rate $(\mathrm{Cr})$. Whale Optimization algorithm is a recently developed metaheuristic algorithm with many good properties like, few parameters; covers broader area in the search space; and exploration of the search space is made efficient by using randomly selected search agent in place of best search agent so far. To achieve balance between exploitation and exploration of the search space, hybridization of DE and WO is proposed; the proposed DEWO algorithm is explained in detail in the next section.

\section{PROPOSED METHODOLOGY}

This section illustrates the methodology adopted in this research work to segment brain MR images. In this study, a 2D histogram is created using gray scale image and non-local means filtered image of the original image. This 2D histogram is used to obtain optimal multilevel thresholds by applying proposed hybrid algorithm DEWO along with Renyi entropy. Threshold levels so obtained are used for the image segmentation process; the segmented and color-mapped images are returned as the final output by the proposed algorithm. The segmented image helps to differentiate between objects and background in the original image. Flowchart of the proposed methodology is illustrated in figure 1.

\section{A Proposed Hybrid Algorithm - DEWO:}

The proposed hybrid algorithm DEWO optimizes the image segmentation method for brain MR images based on 2D histogram thresholding. The proposed hybrid algorithm combines the advantages of better exploitation of DE and better exploration of WO. The algorithm is described as follows:

1. Initialize the population with NP (population size) parameter vectors, making population size for each generation as NP.

2. Set parameters $\mathrm{C}_{\mathrm{R}}, \mathrm{F}, \mathrm{a}$ and $\mathrm{r}$ corresponding to both $\mathrm{DE}$ and $\mathrm{WO}$ algorithms.

3. Calculate the fitness of each individual present in the population.

4. Sort the population in decreasing order of their fitness value (for maximization of fitness function).

5. Repeat steps6-8while maximum iteration is reached or stopping criteria is met.

6. For each individual belonging within range of [1, (NP/2)] of sorted population do - - Choose three different individuals other the one being processed randomly.

$$
X_{r 1} \neq X_{r 2} \neq X_{r 3} \neq X_{j}
$$

- - Apply mutation by adding the weighted difference of the two vectors to the third vector.

$V=X_{r 1}+F *\left(X_{r 2}-X_{r 3}\right)$

where, F: scaling factor

- - Apply Crossover by mixing mutated vector ' $\mathrm{V}$ ' with target vector ' $\mathrm{X}$ '.

$$
U= \begin{cases}V & \text { if rand }_{j} \leq C r \\ X & \text { otherwise }\end{cases}
$$


where, $C r$ : crossover rate

- - Perform selection between the trial vector ' $U$ ' and the target vector ' $X$ ' depending upon which is strictly superior

$X(t+1)= \begin{cases}U & \text { iff }(U) \leq f(X) \\ X & \text { otherwise }\end{cases}$

For each individual belonging within range of $[(\mathrm{NP} / 2)+1, \mathrm{NP}]$ of sorted population do

- Update value of parameters a, r, l, A, C and p. Here $\mathrm{p}$ is the probability to choose between shrinking encircling mechanism or spiral model

- if $\mathrm{p}<0.5$ and $|\mathrm{A}|<1$ then:

where:

A and C: coefficient vectors

$X^{*}(t)$ : Position vector for best solution

- if $\mathrm{p}<0.5$ and $|\mathrm{A}| \geq 1$ then:

where, $X_{r n d}(t):$ Random position vector

- if $\mathrm{p} \geq 0.5$ then:

$D^{\prime}=\left|X^{*}(t)-X(t)\right|$

$\mathrm{X}(\mathrm{t}+1)=\mathrm{D}^{\prime} \cdot \mathrm{e}^{\mathrm{bl}} \cdot \cos (2 \grave{\mathrm{A}})+\mathrm{X}^{*}(\mathrm{t})$

- Check for limit bounds for $\mathrm{X}$ vector.

- Update fitness of each individual in the population range and get best individual.

7. Update for global best solution by comparing each personal best solution of population range.

B Overview Algorithms used for Comparison:

Table 2 describes ABC and CS metaheuristic algorithms used for comparison with the proposed hybrid algorithm. Table 2 shows a Description of Metaheuristic Algorithms.

C Simple Statistics Error and Correlation Based Metrics:

Some of the prominent performance metrics used in this study to evaluate the performance of the segmentation process are described in Table 3 below.

\section{EXPERIMENTAL RESULTS}

The optimal threshold values obtained for brain MR images using the proposed hybrid algorithm, DEWO, were found to give better results for all entropy functions considered in comparison to other metaheuristic algorithms like DE, WO, ABC and CS. MATLAB is used to implement all algorithms and to perform segmentation. Figure 1 shows the original MR image, grayscale image, filtered image and 2D histogram of the MR image. The optimal threshold values and the fitness function value by each algorithm for different entropy functions is illustrated in Table 4. Figures 2 and 3 depict the comparison of the result of all metaheuristic algorithms for Renyi entropy, between-class variance and Tsallis entropy respectively. Tables 4-6 show segmented and color-mapped images obtained after applying threshold values obtained through different metaheuristic algorithms for different entropy functions. Tables 5 and 6 illustrate performance metrics value for all meta heuristic algorithms for different entropy functions. 
Table 2. Description of metaheuristic algorithms

\begin{tabular}{|l|l|l|}
\hline Algorithm & Author/year & Description \\
\hline ABC & $\begin{array}{l}\text { Karaboga, } \\
2007\end{array}$ & $\begin{array}{l}\text { ABC simulates the behavior of real bees for solving multi-dimensional and multimodal } \\
\text { optimization problems. In ABC, bee colony is divided into three groups: the first group } \\
\text { is called employed bees (those which go to find the food source), the second group is } \\
\text { called onlookers (that make the decision to select food source) while the last group is } \\
\text { scouts (that search randomly for the food source). } \\
\text { Position Update: } V=X_{i j}+\varphi *\left(X_{i j}-X_{k j}\right)\end{array}$ \\
\hline CS & $\begin{array}{l}\text { Yang and Deb, } \\
2009\end{array}$ & $\begin{array}{l}\text { In CS algorithm, each set of nests with one egg are placed at random locations in the } \\
\text { algorithm's search space where the egg represents a candidate solution. Search pattern } \\
\text { for discovering best nest is inspired by Levy Flights which is an efficient technique as } \\
\text { compared to other random walks. } \\
\text { Position Update: } x(t+1)=x(t)+a \oplus l e v y(\lambda)\end{array}$ \\
\hline
\end{tabular}

Table 3. Description of performance metrics of segmentation process

\begin{tabular}{|l|l|}
\hline Performance Metrics & Formula \\
\hline Normalized Absolute Error (NAE) & $\mathrm{NAE}=\frac{\sum_{\mathrm{x}=1}^{\mathrm{M}} \sum_{\mathrm{y}=1}^{\mathrm{N}}|\mathrm{X}(\mathrm{x}, \mathrm{y})-\mathrm{Y}(\mathrm{x}, \mathrm{y})|}{\sum_{\mathrm{x}=1}^{\mathrm{M}} \sum_{\mathrm{y}=1}^{\mathrm{N}}|\mathrm{X}(\mathrm{x}, \mathrm{y})|}$ \\
\hline Normalized Cross-Correlation (NCC) & $\mathrm{NCC}=\frac{1}{\mathrm{n}} \sum_{\mathrm{x}, \mathrm{y}} \frac{1}{\tilde{\mathrm{A}}_{\mathrm{x}} \tilde{\mathrm{A}}_{\mathrm{y}}}(\mathrm{X}(\mathrm{x}, \mathrm{y})-\overline{\mathrm{X}})(\mathrm{Y}(\mathrm{x}, \mathrm{y})-\overline{\mathrm{Y}})$ \\
\hline Root Mean Squared Error (RMSE) & $R M S E=\sqrt{E(\hat{\theta}-\theta)^{2} \mid}$ \\
\hline Peak Signal to Noise Ratio (PSNR) & $\mathrm{PSNR}=10 \log _{10} \frac{\left(2^{b}-1\right)^{2}}{\sqrt{M S E}}$ \\
\hline Structural Similarity Index (SSIM) & $\mathrm{SSIM}=\frac{\left(2 \times \overline{\mathrm{X}} \times \overline{\mathrm{Y}}+\mathrm{c}_{1}\right)(2 \times \tilde{\mathrm{A}}}{\left(\tilde{\mathrm{A}}_{\mathrm{x}}^{2}+\tilde{\mathrm{A}}_{\mathrm{y}}^{2}+\mathrm{c}_{2}\right) \times\left((\overline{\mathrm{X}})^{2}+(\overline{\mathrm{Y}})^{2}+\mathrm{c}_{1}\right)}$ \\
\hline
\end{tabular}

\section{CONCLUSION}

This study proposes a hybrid metaheuristic algorithm, DEWO, by combining DE algorithm with WO algorithm for multilevel image segmentation using non-local mean 2D histogram thresholding method. In this paper, we have performed 3-level thresholding to segment brain MR images for detection of tumors. Results show that the proposed algorithm performs better than other metaheuristic algorithms like DE, WO, ABC and CS.This comparison was performed for different entropy-based functions and the proposed hybrid algorithm outperformed other algorithms in all scenarios. Recently developed WO algorithm when combined with $\mathrm{DE}$ algorithm is able to provide a better balance between exploration 


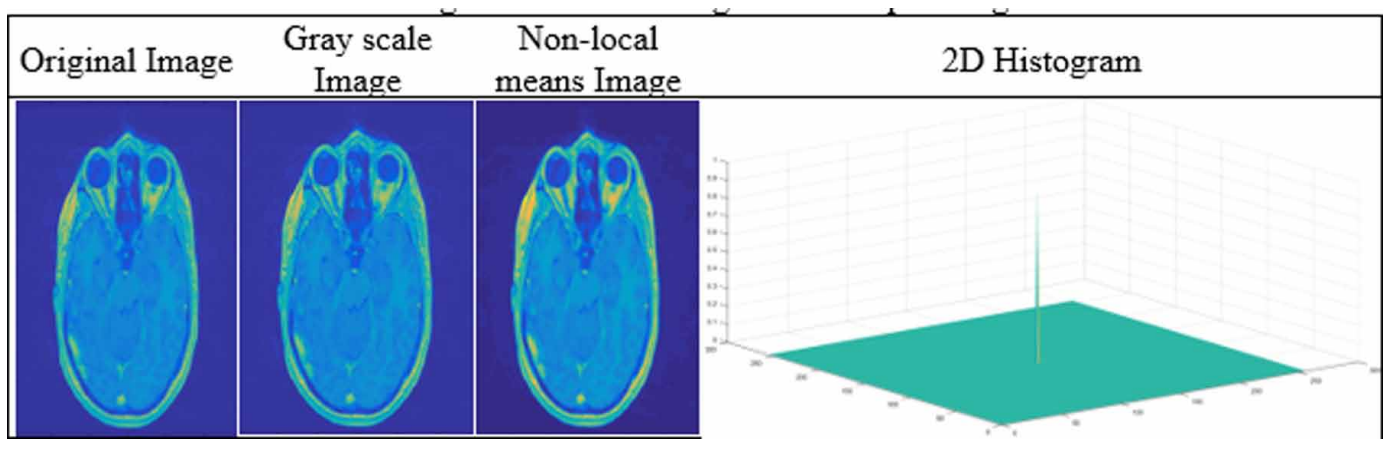

Table 4. Optimal thresholds and fitness function value for different entropy functions

\begin{tabular}{|l|l|l|l|l|l|l|}
\hline & \multicolumn{2}{|l|}{ Renyi Entropy } & \multicolumn{2}{l|}{ Between-class Variance } & \multicolumn{2}{l|}{ Tsallis Entropy } \\
\hline Algorithm & $\begin{array}{l}\text { Optimal } \\
\text { Thresholds } \\
\text { T1, T2, T3 }\end{array}$ & $\begin{array}{l}\text { Optimum } \\
\text { Value }\end{array}$ & $\begin{array}{l}\text { Optimal } \\
\text { Thresholds } \\
\text { T1, T2, T3 }\end{array}$ & $\begin{array}{l}\text { Optimum } \\
\text { Value }\end{array}$ & $\begin{array}{l}\text { Optimal } \\
\text { Thresholds } \\
\text { T1, T2, T3 }\end{array}$ & $\begin{array}{l}\text { Optimum } \\
\text { Value }\end{array}$ \\
\hline DE & $1,129,213$ & 1.1951 & $144,147,156$ & 15972.44 & $1,129,255$ & 1.0915 \\
\hline ABC & $25,129,188$ & 1.1951 & $146,160,251$ & 15972.44 & $129,145,160$ & 1.6372 \\
\hline CS & $129,132,149$ & 1.7926 & $153,156,248$ & 15972.44 & 1,129236 & 1.0915 \\
\hline WO & $1,75,129$ & 0.59755 & $6,70,232$ & 82251.04 & $39,107,137$ & 0.47603 \\
\hline DEWO & $129,138,255$ & 1.7928 & $138,199,209$ & 16852.74 & $129,138,255$ & 1.6372 \\
\hline
\end{tabular}

Table 5. Performance metrics for Renyi entropy

\begin{tabular}{|l|l|l|l|l|l|}
\hline Algorithm & NAE & NCC & RMSE & PSNR & SSIM \\
\hline DE & 1 & 32767 & 1 & -22.8807 & 6 \\
\hline ABC & 1 & 32767 & 1 & -22.8807 & 6 \\
\hline CS & 1 & 32767 & 1 & -22.8807 & 8 \\
\hline WO & 1 & 32767 & 2 & -25.8910 & 4 \\
\hline DEWO & 1 & 32767 & 1 & -22.8807 & 8 \\
\hline
\end{tabular}

and exploitation of the search space. Regardless of the entropy function chosen, our algorithm is able to achieve maximized value of fitness function than any other algorithm being compared. Performance metrics of segmented image with the original image show that there is not much degradation on the quality of the image after the segmentation of brain MR images. The proposed algorithm is able to detect tumors in a far more clear and accurate form than the other methods.

For future work, WO and DE algorithms could be hybridized with different population-based and local search-based algorithms to see interesting results. Multilevel image segmentation can be combined with morphological processing and image subtraction; thereby results could be further analyzed. Further, the proposed hybrid algorithm can be applied to many more real-world problems like fault detection, habitat monitoring etc. 
Table 6. Performance metrics for Tsallis entropy and between-class variance

\begin{tabular}{|l|l|l|l|l|l|l|l|l|l|l|}
\hline & \multicolumn{4}{|l}{ Tsallis Entropy } & \multicolumn{3}{l|}{ Between-class Variance } \\
\hline Algorithm & NAE & NCC & RMSE & PSNR & SSIM & NAE & NCC & RMSE & PSNR & SSIM \\
\hline DE & 1 & 32767 & 2 & -25.8910 & 8 & 1 & 32767 & 1 & -22.8807 & 6 \\
\hline ABC & 1 & 32767 & 1 & -22.8807 & 6 & 1 & 32767 & 1 & -22.8807 & 6 \\
\hline CS & 1 & 32767 & 2 & -25.8910 & 5 & 1 & 32767 & 1 & -22.8807 & 6 \\
\hline WO & 1 & 32767 & 2 & -25.8910 & 6 & 1 & 32767 & 1 & -22.8807 & 4 \\
\hline DEWO & 1 & 32767 & 1 & -22.8807 & 8 & 1 & 32767 & 1 & -22.8807 & 7 \\
\hline
\end{tabular}

Figure 2. Segmented and color-mapped image for Tsallis entropy

\begin{tabular}{|c|c|c|c|c|c|}
\hline $\begin{array}{l}\text { Algorith } \\
\text { ms }\end{array}$ & $\begin{array}{l}\text { Segmented } \\
\text { Image }\end{array}$ & $\begin{array}{l}\text { Color-mapped } \\
\text { Image }\end{array}$ & \begin{tabular}{|c|}
$\begin{array}{c}\text { Algorith } \\
\text { ms }\end{array}$ \\
\end{tabular} & Segmented Image & $\begin{array}{c}\text { Color-mapped } \\
\text { Image }\end{array}$ \\
\hline $\mathrm{DE}$ & & & WO & & \\
\hline $\mathrm{ABC}$ & & & DEWO & & \\
\hline $\mathrm{CS}$ & & & & & \\
\hline
\end{tabular}


Figure 3. Segmented and color-mapped image of Renyi Entropy, segmented and color-mapped image of between-class variance
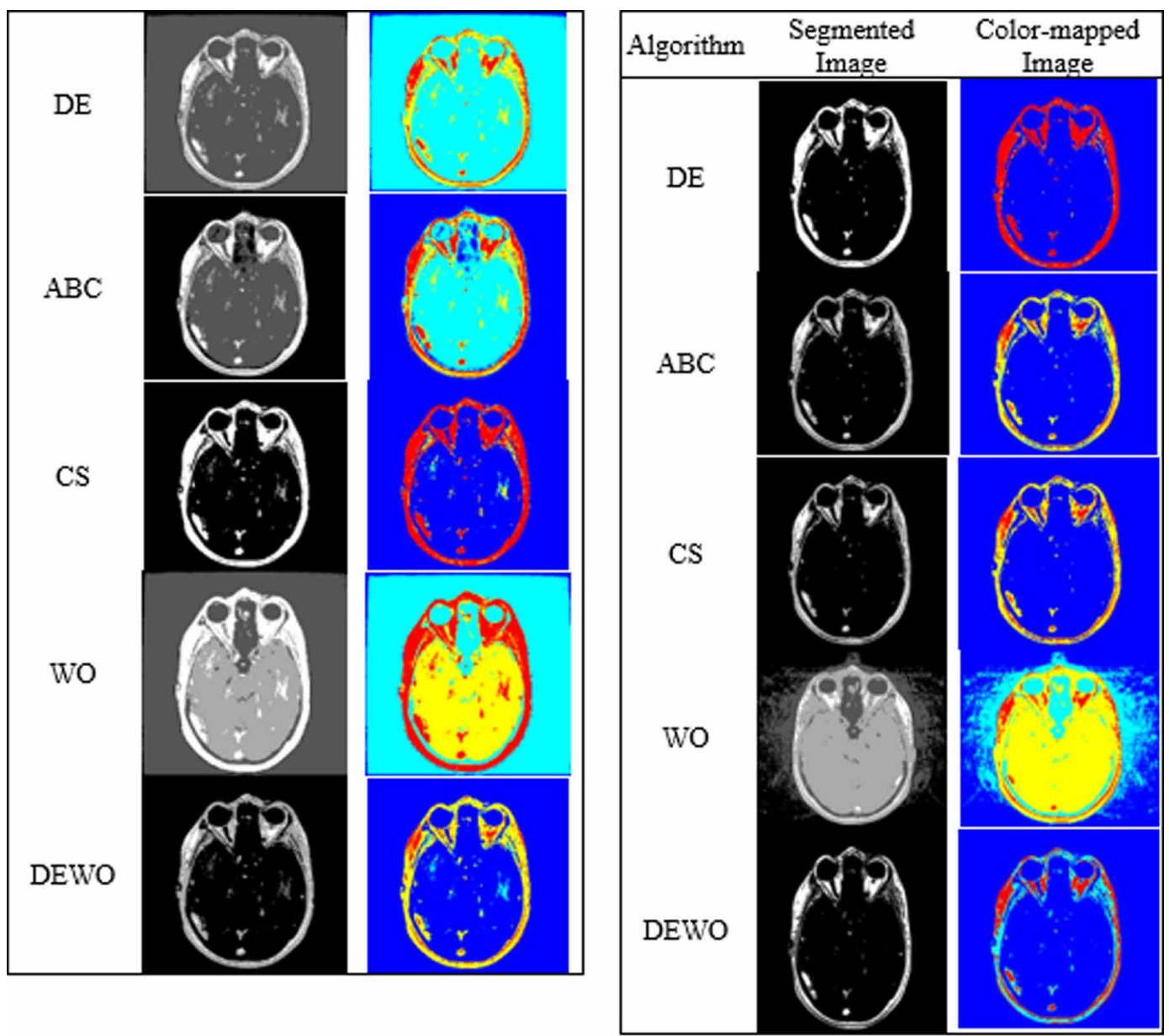


\section{REFERENCES}

Abdeldaim, A. M., Sahlol, A. T., Elhoseny, M., \& Hassanien, A. E. (2018). Computer-aided acute lymphoblastic leukemia diagnosis system based on image analysis. In Advances in Soft Computing and Machine Learning in Image Processing (pp. 131-147). Cham: Springer. doi:10.1007/978-3-319-63754-9_7

Abutaleb, A. S. (1989). Automatic thresholding of gray-level pictures using two-dimensional entropy. Computer Vision Graphics and Image Processing, 47(1), 22-32. doi:10.1016/0734-189X(89)90051-0

Al-Tarawneh, M. S. (2012). Lung cancer detection using image processing techniques. Leonardo Electronic Journal of Practices and Technologies, 11(21), 147-158.

Bahadure, N. B., Ray, A. K., \& Thethi, H. P. (2017). Image analysis for MRI based brain tumor detection and feature extraction using biologically inspired BWT and SVM. International Journal of Biomedical Imaging. PMID:28367213

Dawngliana, M., Deb, D., Handique, M., \& Roy, S. (2015, September). Automatic brain tumor segmentation in MRI: Hybridized multilevel thresholding and level set. Proceedings of the 2015 International Symposium on Advanced Computing and Communication (ISACC) (pp. 219-223). IEEE. doi:10.1109/ISACC.2015.7377345

Feng, Y., Zhao, H., Li, X., Zhang, X., \& Li, H. (2017). A multi-scale 3D Otsu thresholding algorithm for medical image segmentation. Digital Signal Processing, 60, 186-199. doi:10.1016/j.dsp.2016.08.003

Fengjie, S., He, W., \& Jieqing, F. (2009, May). 2D Otsu segmentation algorithm based on simulated annealing genetic algorithm for iced-cable images. Proceedings of the 2009 International Forum on Information Technology and Applications (Vol. 2, pp. 600-602). IEEE.

Heimann, T., Van Ginneken, B., Styner, M. A., Arzhaeva, Y., Aurich, V., Bauer, C., \& Bello, F. et al. (2009). Comparison and evaluation of methods for liver segmentation from CT datasets. IEEE Transactions on Medical Imaging, 28(8), 1251-1265. doi:10.1109/TMI.2009.2013851 PMID:19211338

Horng, M. H., \& Liou, R. J. (2011). Multilevel minimum cross entropy threshold selection based on the firefly algorithm. Expert Systems with Applications, 38(12), 14805-14811. doi:10.1016/j.eswa.2011.05.069

Jubany, I., Lafuente, J., Carrera, J., \& Baeza, J. A. (2009). Automated thresholding method (ATM) for biomass fraction determination using FISH and confocal microscopy. Journal of Chemical Technology \& Biotechnology, 84(8), 1140-1145.

Kapur, J. N., Sahoo, P. K., \& Wong, A. K. (1985). A new method for gray-level picture thresholding using the entropy of the histogram. Computer Vision Graphics and Image Processing, 29(3), 273-285. doi:10.1016/0734189X(85)90125-2

Karaboga, D., \& Basturk, B. (2007). A powerful and efficient algorithm for numerical function optimization: Artificial bee colony (ABC) algorithm. Journal of Global Optimization, 39(3), 459-471. doi:10.1007/s10898007-9149-x

Kittler, J., \& Illingworth, J. (1986). Minimum error thresholding. Pattern Recognition, 19(1), 41-47. doi:10.1016/0031-3203(86)90030-0

Kumar, S., Sharma, T. K., Pant, M., \& Ray, A. K. (2012). Adaptive artificial bee colony for segmentation of CT lung images. Int. J. Comp. App. iRAFIT, 5, 1-5.

Lee, L. K., Liew, S. C., \& Thong, W. J. (2015). A review of image segmentation methodologies in medical image. In Advanced computer and communication engineering technology (pp. 1069-1080). Cham: Springer. doi:10.1007/978-3-319-07674-4_99

Li, Y., Jiao, L., Shang, R., \& Stolkin, R. (2015). Dynamic-context cooperative quantum-behaved particle swarm optimization based on multilevel thresholding applied to medical image segmentation. Information Sciences, 294, 408-422. doi:10.1016/j.ins.2014.10.005

Maulik, U. (2009). Medical image segmentation using genetic algorithms. IEEE Transactions on Information Technology in Biomedicine, 13(2), 166-173. doi:10.1109/TITB.2008.2007301 PMID:19272859

Mirjalili, S., \& Lewis, A. (2016). The whale optimization algorithm. Advances in Engineering Software, 95 , 51-67. doi:10.1016/j.advengsoft.2016.01.008 
Mittal, H., \& Saraswat, M. (2018). An optimum multi-level image thresholding segmentation using non-local means 2D histogram and exponential Kbest gravitational search algorithm. Engineering Applications of Artificial Intelligence, 71, 226-235. doi:10.1016/j.engappai.2018.03.001

Mohammed, M. A., Ghani, M. K. A., Hamed, R. I., Abdullah, M. K., \& Ibrahim, D. A. (2017). Automatic segmentation and automatic seed point selection of nasopharyngeal carcinoma from microscopy images using region growing based approach. Journal of Computational Science, 20, 61-69.

Nakib, A., Roman, S., Oulhadj, H., \& Siarry, P. (2007, August). Fast brain MRI segmentation based on twodimensional survival exponential entropy and particle swarm optimization. Proceedings of the 200729 th Annual International Conference of the IEEE Engineering in Medicine and Biology Society (pp. 5563-5566). IEEE Press. doi:10.1109/IEMBS.2007.4353607

Namburu, A., Samay, S., \& Edara, S. R. (2017). Soft fuzzy rough set-based MR brain image segmentation. Applied Soft Computing, 54, 456-466. doi:10.1016/j.asoc.2016.08.020

Oh, W., \& Lindquist, B. (1999). Image thresholding by indicator kriging. IEEE Transactions on Pattern Analysis and Machine Intelligence, 21(7), 590-602. doi:10.1109/34.777370

Otsu, N. (1979). A threshold selection method from gray-level histograms. IEEE Transactions on Systems, Man, and Cybernetics, 9(1), 62-66. doi:10.1109/TSMC.1979.4310076

Otsu, N. (1979). A threshold selection method from gray-level histograms. IEEE Transactions on Systems, Man, and Cybernetics, 9(1), 62-66. doi:10.1109/TSMC.1979.4310076

Panda, R., Agrawal, S., Samantaray, L., \& Abraham, A. (2017). An evolutionary gray gradient algorithm for multilevel thresholding of brain MR images using soft computing techniques. Applied Soft Computing, 50, 94-108. doi:10.1016/j.asoc.2016.11.011

Panda, R., Agrawal, S., Samantaray, L., \& Abraham, A. (2017). An evolutionary gray gradient algorithm for multilevel thresholding of brain MR images using soft computing techniques. Applied Soft Computing, 50, 94-108. doi:10.1016/j.asoc.2016.11.011

Pun, T. (1980). A new method for grey-level picture thresholding using the entropy of the histogram. Signal Processing, 2(3), 223-237. doi:10.1016/0165-1684(80)90020-1

Raja, N. S. M., Fernandes, S. L., Dey, N., Satapathy, S. C., \& Rajinikanth, V. (2018). Contrast enhanced medical MRI evaluation using Tsallis entropy and region growing segmentation. Journal of Ambient Intelligence and Humanized Computing, 1-12.

Rajinikanth, V., Satapathy, S. C., Fernandes, S. L., \& Nachiappan, S. (2017). Entropy based segmentation of tumor from brain MR images-a study with teaching learning based optimization. Pattern Recognition Letters, 94, 87-95. doi:10.1016/j.patrec.2017.05.028

Roopini, I. T., Vasanthi, M., Rajinikanth, V., Rekha, M., \& Sangeetha, M. (2018). Segmentation of tumor from brain MRI using fuzzy entropy and distance regularised level set. In Computational Signal Processing and Analysis (pp. 297-304). Singapore: Springer. doi:10.1007/978-981-10-8354-9_27

Sahoo, P. K., \& Arora, G. (2004). A thresholding method based on two-dimensional Renyi's entropy. Pattern Recognition, 37(6), 1149-1161. doi:10.1016/j.patcog.2003.10.008

Saleh, M. D., \& Eswaran, C. (2012). An efficient algorithm for retinal blood vessel segmentation using h-maxima transform and multilevel thresholding. Computer Methods in Biomechanics and Biomedical Engineering, 15(5), 517-525. doi:10.1080/10255842.2010.545949 PMID:21331960

Sarkar, S., \& Das, S. (2013). Multilevel image thresholding based on 2D histogram and maximum Tsallis entropy-A differential evolution approach. IEEE Transactions on Image Processing, 22(12), 4788-4797. doi:10.1109/TIP.2013.2277832 PMID:23955760

Sarkar, S., Das, S., \& Chaudhuri, S. S. (2017). Multi-level thresholding with a decomposition-based multiobjective evolutionary algorithm for segmenting natural and medical images. Applied Soft Computing, 50, 142-157. doi:10.1016/j.asoc.2016.10.032 
Sathya, P. D., \& Kayalvizhi, R. (2011). Modified bacterial foraging algorithm based multilevel thresholding for image segmentation. Engineering Applications of Artificial Intelligence, 24(4), 595-615. doi:10.1016/j. engappai.2010.12.001

Sezgin, M., \& Sankur, B. (2004). Survey over image thresholding techniques and quantitative performance evaluation. Journal of Electronic Imaging, 13(1), 146-166. doi:10.1117/1.1631315

Shahdoosti, H. R., \& Tabatabaei, Z. (2019). MRI and PET/SPECT image fusion at feature level using ant colony based segmentation. Biomedical Signal Processing and Control, 47, 63-74. doi:10.1016/j.bspc.2018.08.017

Sieracki, M. E., Reichenbach, S. E., \& Webb, K. L. (1989). Evaluation of automated threshold selection methods for accurately sizing microscopic fluorescent cells by image analysis. Applied and Environmental Microbiology, 55(11), 2762-2772. PMID:2516431

Storn, R., \& Price, K. (1997). Differential evolution-a simple and efficient heuristic for global optimization over continuous spaces. Journal of Global Optimization, 11(4), 341-359. doi:10.1023/A:1008202821328

Sumathi, R., Venkatesulu, M., \& Arjunan, S. P. (2018). Extracting tumor in MR brain and breast image with Kapur's entropy based Cuckoo Search Optimization and morphological reconstruction filters. Biocybernetics and Biomedical Engineering, 38(4), 918-930. doi:10.1016/j.bbe.2018.07.005

Tang, Y.G., Di Qiu-Yan, Z.L.X., Guan, X.P., \& Liu, F.C. (2009). Image thresholding segmentation based on two-dimensional minimum Tsallis-cross entropy. Acta Physica Sinica.

Wang, X. N., Feng, Y. J., \& Feng, Z. R. (2005, August). Ant colony optimization for image segmentation. Proceedings of 2005 International Conference on Machine Learning and Cybernetics, 2005. (Vol. 9, pp. 53555360). IEEE Press.

Wong, A. K., \& Sahoo, P. K. (1989). A gray-level threshold selection method based on maximum entropy principle. IEEE Transactions on Systems, Man, and Cybernetics, 19(4), 866-871. doi:10.1109/21.35351

Xiao-Feng, L. I., Hui-Ying, L. I. U., Ming, Y., \& Tai-Ping, W. E. I. (2016). Infrared image segmentation based on AAFSA and 2D-Renyi entropy threshold selection. DEStech Transactions on Computer Science and Engineering.

Yang, X. S., \& Deb, S. (2009, December). Cuckoo search via Lévy flights. Proceedings of the 2009 World Congress on Nature \& Biologically Inspired Computing (NaBIC) (pp. 210-214). IEEE. doi:10.1109/NABIC.2009.5393690

Yeo, S. Y., Romero, J., Loper, M., Machann, J., \& Black, M. (2018). Shape estimation of subcutaneous adipose tissue using an articulated statistical shape model. Computer Methods in Biomechanics and Biomedical Engineering. Imaging \& Visualization, 6(1), 51-58. doi:10.1080/21681163.2016.1163508

Zhu, H., Zhuang, Z., Zhou, J., Zhang, F., Wang, X., \& Wu, Y. (2017). Segmentation of liver cyst in ultrasound image based on adaptive threshold algorithm and particle swarm optimization. Multimedia Tools and Applications, 76(6), 8951-8968. doi:10.1007/s11042-016-3486-z

Sumit Kumar, Dr., is Associate Professor in Department of Computer Science and Engineering, Amity University, Uttar Pradesh, Noida. His area of interest includes software testing, machine learning and metaheuristic algorithms.

Garima Vig is a research scholar of Computer Science Engineering Department, Amity University, Uttar Pradesh, NOIDA. Her area of interest includes metaheuristic algorithms, image processing and machine learning.

Sapna Varshney has a keen interest in research and teaching. Her area of interest includes metaheuristic optimization, software engineering, and data mining.

Priti Bansal has a PhD from Delhi University. Her area of interest includes software testing, metaheuristic algorithms, machine learning, and image processing. 